\title{
压电复合材料用于透镜式聚焦换能器的研究
}

何 敏 ${ }^{1}$, 郝 琦 $^{1}$, 王科金金 ${ }^{1}$, 曾德平 ${ }^{1}$, 叶方伟 ${ }^{2}$, 李发琪 ${ }^{1}$, 王智彪 ${ }^{1}$, 何虹莹 ${ }^{3}$

(1. 重庆医科大学生物医学工程学院, 省部共建超声医学工程国家重点实验室培育基地一重庆市超声医学工程重 点实验室, 超声医学工程重庆市重点实验室, 重庆 400016; 2. 超声医疗国家工程研究中心, 重庆 401121; 3. 四川 大学 材料科学与工程学院, 成都 610064)

摘 要: 为了增加聚焦换能器的带宽, 抑制其多模振动耦合现象, 提高电声转换效率, 实验采用 1-3型压电复合材料 代替压电陶瓷作为超声发射材料, 设计并制作了一种新型的 1-3 型压电复合材料透镜式聚焦换能器。通过频率特性 的对比研究, 证明了 1-3 型压电复合材料透镜式聚焦换能器不仅能增加换能器的带宽, 达到 PZT 压电陶瓷透镜式聚 焦换能器带宽的 3.13 倍, 而且能明显抑制径向振动, 得到单一纯净的厚度振动模态。另外, 1-3 型压电复合材料透 镜式聚焦换能器的电声转换效率达到 PZT 压电陶瓷透镜式聚焦换能器的 1.88 倍, 为高效率聚焦超声换能器的实现 提供了理论及实验基础。

关 键 词: 1-3 型压电复合材料; 聚焦换能器; 声透镜; 电声转换

中图分类号: TQ050 文献标识码: A

\section{Properties of a Lens-focused Transducer Based on Piezoelectric Composites}

\author{
HE Min ${ }^{1}$, HAO Qi ${ }^{1}$, WANG Ke-Xin ${ }^{1}$, ZENG De-Ping ${ }^{1}$, YE Fang-Wei ${ }^{2}$, LI Fa-Qi ${ }^{1}$, WANG Zhi-Biao ${ }^{1}$, HE Hong-Ying ${ }^{3}$
}

(1. State Key Laboratory of Ultrasound Engineering in Medicine Co-founded by Chongqing and the Ministry of Science and Technology, College of Biomedical Engineering, Chongqing Key Laboratory of Biomedical Engineering, Chongqing Medical University, Chongqing 400016, China; 2. National Engineering Research Center of Ultrasound Medicine, Chongqing 401121, China; 3. College of Materials Science and Engineering of Sichuan University, Chengdu, Sichuan 610064, China)

\begin{abstract}
To broaden the bandwidth of focused transducer with suppressing the multimode coupling phenomenon, and improving the electro-acoustic conversion efficiency, 1-3 piezoelectric composites as the ultrasonic emission material was used to replace Pb-based lanthanum doped zirconate titanates ceramic. A new type of lens-focused transducer was designed and produced based on 1-3 piezoelectric composites. Through the comparative study of frequency characteristic, it is proved that the 1-3 piezoelectric composites lens-focused transducer can not only increase the bandwidth of the transducer which is 3.13 times of the one based on Pb-based lanthanum doped zirconate titanates, but also suppress the radial vibration obviously to obtain a single pure vibration modes. Besides, the electro-acoustic conversion efficiency of the former lens-focused transducer is 1.88 times of the latter one. These results provide a theoretical and experimental foundation for the realization of ultrasonic transducer of high efficiency on reliability and stability.
\end{abstract}

Key words: 1-3 piezoelectric composites; focused transducer; acoustic lens; electro-acoustic conversion

收稿日期：2014-11-20; 收到修改稿日期：2015-03-06

基金项目: 国家自然科学基金委重大科学仪器设备研制专项(81127901); 国家自然科学基金(81201102, 11274404);

国家重点基础研究发展计划(973 项目, 2011CB707900); 重庆市科技项目(2010BB5368)

National Natural Science Foundation of China (81127901, 81201102, 11274404); State Key Development Program for

Basic Research of China (973 program, 2011CB707900); Science and Technology Project of Chongqing (2010BB5368)

作者简介：何 敏(1990-), 女, 硕士研究生. E-mail:kathy1210@foxmail.com

通讯作者: 曾德平, 副教授. E-mail: zengdp@haifu.com.cn 
随着高强度聚焦超声(high intensity focused ultrasound, 简记为 HIFU)在临床治疗中的成功应用, 医 用超声技术逐渐从检测领域拓展到治疗领域, 被喻为 21 世纪的“科技前沿”, [1]。作为聚焦超声技术的核心器 件 ${ }^{[2]}$, 目前普遍使用的透镜式聚焦换能器是用压电陶 瓷制作的, 它主要利用压电陶瓷的厚度振动来实现电 声能量转换。但是, 由于压电陶瓷存在多振动模耦合 的问题, 使得该类型换能器的电声转换效率较低。

近年来, 换能器材料的研究取得良好的进展, 如 压电复合材料在频率带宽、低阻抗和电声转换效率等 方面表现出优势，已逐步走向应用。1-3 型压电复合材 料是由一维连通的压电相平行排列于三维连通的聚 合物中而形成的两相压电复合材料, 它不仅保留了压 电陶瓷的压电性和刚度, 还增强了其各向异性和纵向 耦合能力。最近汤旭东等 ${ }^{[3]}$ 开展了基于 1-3 型压电复 合材料的透镜式聚焦换能器的仿真研究, 认为该材料 在抑制压电陶瓷的径向振动耦合方面有着良好的性 能。但该材料在透镜式聚焦换能器的实际应用则少有 报道。在汤旭东仿真设计的基础上, 本工作采用相同 的铅钛酸铅(Pb-based lanthanum doped zirconate titanates, PZT)压电陶瓷，一个直接制作成 PZT 压电陶瓷 透镜式聚焦换能器, 另一个用 “切割-填充法”制备成 1-3 型压电复合材料, 再制作 1-3 型压电复合材料透镜 式聚焦换能器。对比研究了这两种透镜式聚焦换能器 的频率特性和电声转换效率。

\section{1 实验原理}

美国学者 Newnham 等 ${ }^{[4]}$ 于 20 世纪 70 年代末首次 提出了 1-3 型压电复合材料的概念。1-3 型压电复合材 料是由一维连接的压电陶瓷相周期性地排列在三维连 接的聚合物环氧树脂中, 如图 1 所示。压电复合材料 是由两相材料构成, 增加了材料的复杂性, 使得材料 结构和性能之间的关系变得复杂。由于聚合物的绝缘 性, 特殊的力学性能, 良好的韧性等特点 ${ }^{[5-8]}$, 克服了 压电陶瓷在弹性和脆性等方面的缺点, 增加了复合材 料的弹性柔顺性及纵向耦合能力。利用 1-3 型压电复 合材料制备换能器可增加带宽, 降低声阻抗, 更易与

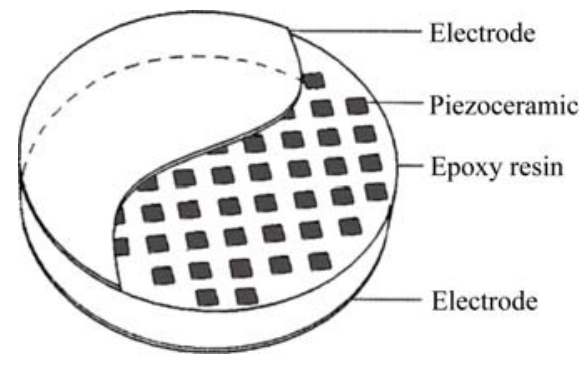

图 1 1-3 型压电复合材料示意图

Fig. 1 Schematic diagram of 1-3 piezoelectric composites

人体组织相匹配; 通过合理调控压电陶瓷与环氧树脂 的体积比还可有效提高换能器的电声转换效率 ${ }^{[9-12]}$ 。

声波在 1-3 型压电复合材料的传播在 20 世纪 90 年代创建的微观力学模型中已有理论解释, 由 Schulgasser ${ }^{[13]}$, Benveniste 和 Dvorak ${ }^{[14]}$ 研究发现的弹 性纤维理论模型, 可以得到 1-3 型纤维压电复合材料 有效模量之间的关系。Dunn ${ }^{[15]}$ 创建的 1-3 型压电复 合材料的微观力学模型, 解释了电弹行为之间的耦 合关系。由于 PZT 压电陶瓷内部各向异性小, 且其 振动时产生的横向分量较大, 使得径向振动明显, 电声转换效率较低。而压电复合材料是由两相材料 构成, PZT 压电陶瓷和聚合物的热膨胀系数不匹配, 导致了两相均衡温度的变化, 从而在两相之间形成 了内部压力。随着内部压力的积累, 由此而产生的电 荷增加, 复合材料内部出现极化, 这一极化分量与 PZT 压电陶瓷的横向分量方向相反, 从而使得 PZT 压电陶瓷的横向分量减弱，径向振动模态被抑制。

\section{2 实验方法}

\subsection{1-3 型压电复合材料的制作}

本研究 1-3 型压电复合材料采用“切割-填充法, [16] 制备。首先, 沿着与 PZT 压电陶瓷块极化轴互相垂直 的两个方向进行准确的切割, 切割出设计要求的槽。然 后将双酚 $\mathrm{A}$ 型环氧树脂与甲基四氢苯酮固化剂按 1:1 的质量比混合, 混合均匀后将其浇注在压电陶瓷的凹 槽里, 常温凝固 $0.5 \mathrm{~h}$ 后, 在 $80^{\circ} \mathrm{C}$ 烘箱中软固化 $10 \mathrm{~h}$ 后 再升温至 $100^{\circ} \mathrm{C}$ 固化 $8 \mathrm{~h}$ 。经磁控溅射 ${ }^{[17]}$ 电极后形成 1-3 型压电复合材料, 制备的基本流程如图 2 所示。
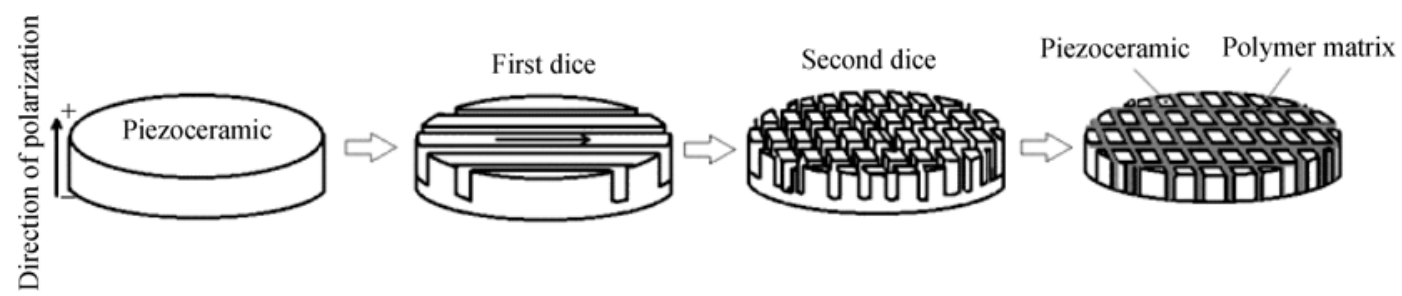

图 2 压电复合材料制作流程图

Fig. 2 Fabrication flow chart of piezoelectric composites 
采用 “切割-填充法”工艺可以灵活地控制压电 陶瓷柱的大小, 从而设计压电复合材料的性能, 这 为将来大功率高强度聚焦超声换能器的制备提供了 可靠的材料基础。图 3 是体式显微镜下观察实验制 得的 1-3 型压电复合材料的实物图片, 其中, 深色 部分为压电陶瓷柱, 浅色部分为环氧树脂。陶瓷柱 尺寸为 $1 \mathrm{~mm} \times 1 \mathrm{~mm} \times 5 \mathrm{~mm}$ (长×宽 $\times$ 高), 陶瓷柱 间距为 $0.5 \mathrm{~mm}$, 压电陶瓷体积分数为 $42.8 \%$ 。

\section{2 换能器的设计和制作}

本实验采用铝透镜作为声波会聚透镜, 因为声波 在铝透镜中传播速度较快, 从而可以减少相差和传播 损失 ${ }^{[18-19]}$ 。换能器结构及各参数如图 4(a)所示, 实物如

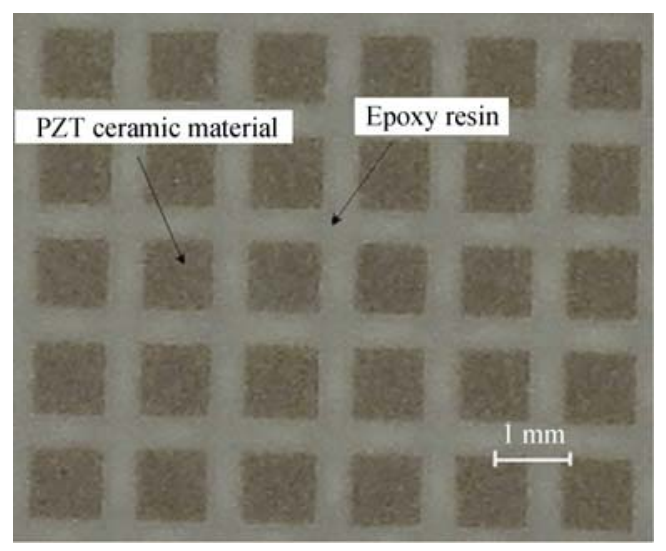

图 3 1-3 型压电复合材料显微镜图片

Fig. 3 Microscope figure of 1-3 piezoelectric composites

(a)
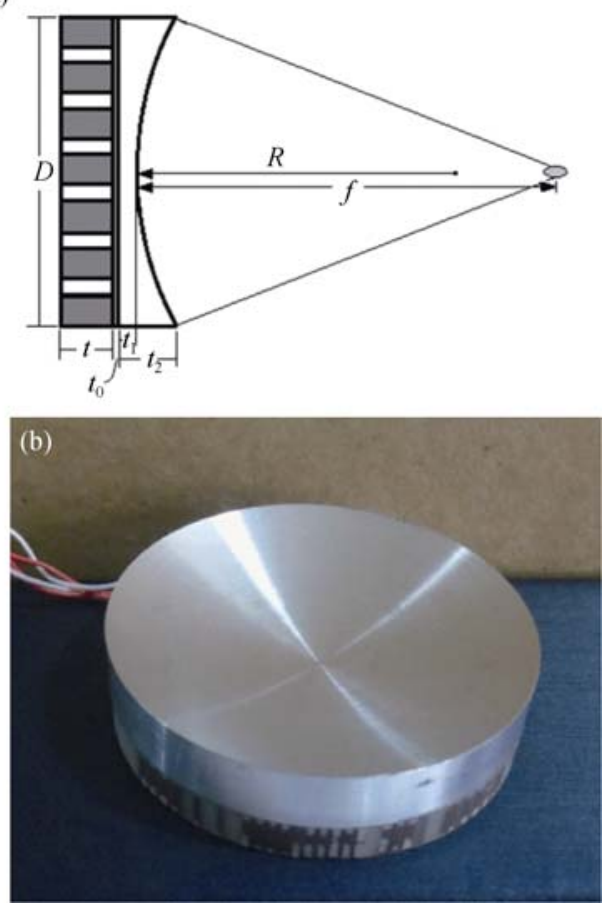

图 4 透镜式聚焦换能器结构示意图(a)和实物图片(b)

Fig. 4 Structure diagram (a) and photo (b) of lens-focused transducer
图 4(b)所示。其中, 1-3 型压电复合材料直径 $D=50 \mathrm{~mm}$, 厚度 $t=5 \mathrm{~mm}$, 粘结层厚度 $t_{0}=0.1 \mathrm{~mm}$ 。铝透镜按如下方 式设计: 中心厚度 $t_{1}=\lambda / 8$ ( $\lambda$ 是超声波波长), 边缘厚度 $t_{2}=8.9 \mathrm{~mm}$, 曲率半径 $R=65.8 \mathrm{~mm}$ 。

$f$ 是换能器的焦距, 凹球面声透镜的焦距由下 列公式计算确定:

$$
\begin{gathered}
f=\frac{R}{1-1 / n} \\
n=\frac{C_{1}}{C_{2}}
\end{gathered}
$$

其中, $R$ 为声透镜中心的曲率半径, $C_{1}$ 为声波在声透 镜中的声速 $\left(C_{1}=6305 \mathrm{~m} / \mathrm{s}\right), C_{2}$ 为声波在媒质中的传 播速度 $\left(C_{2}=1450 \mathrm{~m} / \mathrm{s}\right)$ 。

\section{3 结果与讨论}

采用Agilent 4294A 阻抗分析仪分别对 PZT压电陶 瓷和 1-3 型压电复合材料的阻抗谱进行了测试, 结果如 图 5 所示。图 5(a)、(c)分别是 PZT 压电陶瓷和 1-3 型 压电复合材料的频率-阻抗特性曲线, 对比两图可以看 到, 1-3 型压电复合材料厚度振动模态纯净。PZT 压电 陶瓷厚度振动模态下, 谐振频率 $f_{\mathrm{r}}=437.00 \mathrm{kHz}$, 反谐 振频率 $f_{\mathrm{a}}=440.00 \mathrm{kHz} ; 1-3$ 型压电复合材料的厚度振 动模态下, 谐振频率 $f_{\mathrm{r}}=360.00 \mathrm{kHz}$, 反谐振频率 $f_{\mathrm{a}}=379.00 \mathrm{kHz}$ 。材料频率带宽由 $\Delta f=f_{\mathrm{a}}-f_{\mathrm{r}}$ 确定, 因此 PZT 压电陶瓷的带宽 $\Delta f_{\mathrm{PZT}}=3 \mathrm{kHz}, 1-3$ 型压电 复合材料带宽 $\Delta f_{1-3}=19 \mathrm{kHz}$, 可见在厚度振动模态 下, 1-3 型压电复合材料的带宽比 PZT 压电陶瓷明显 增加。图 5(b)、(d)分别是 PZT 压电陶瓷和 1-3 型压 电复合材料的频率-相位特性曲线, 两者的频率 - 相 位特性曲线也充分证明了 1-3 型压电复合材料可以 有效地抑制多模振动现象。

根据 Guruaja 等 ${ }^{[20]}$ 的研究, 材料的厚度机电耦合 系数 $k_{\mathrm{t}}$ 和径向机电耦合系数 $k_{\mathrm{p}}$ 可由以下公式求得:

$$
\begin{aligned}
& k_{\mathrm{t}}^{2}=\frac{\pi}{2} \frac{f_{\mathrm{t}}}{f_{\mathrm{a}}} \tan \left(\frac{\pi}{2} \frac{\Delta f}{f_{\mathrm{a}}}\right) \\
& k_{\mathrm{p}}{ }^{2}=\frac{\Delta f}{f_{\mathrm{r}}}\left[\frac{R_{1}^{2}-\left(1-\sigma^{2}\right)}{1+\sigma}\right]
\end{aligned}
$$

式中, $f_{\mathrm{t}}$ 是材料的厚度谐振频率, $f_{\mathrm{a}}$ 是材料的厚度反 谐振频率, $f_{\mathrm{r}}$ 是材料的径向谐振频率, $\sigma$ 是材料的泊 松比, 由于 PZT 压电陶瓷和环氧树脂的泊松比均约 为 0.3, 因此 1-3 型压电复合材料的泊松比也近似为 0.3 。 $R 1$ 是材料等效电路串联支路的电阻, 本实验条 件下, $R_{1}=2.049$, 测得的材料参数如表 1 所示。 

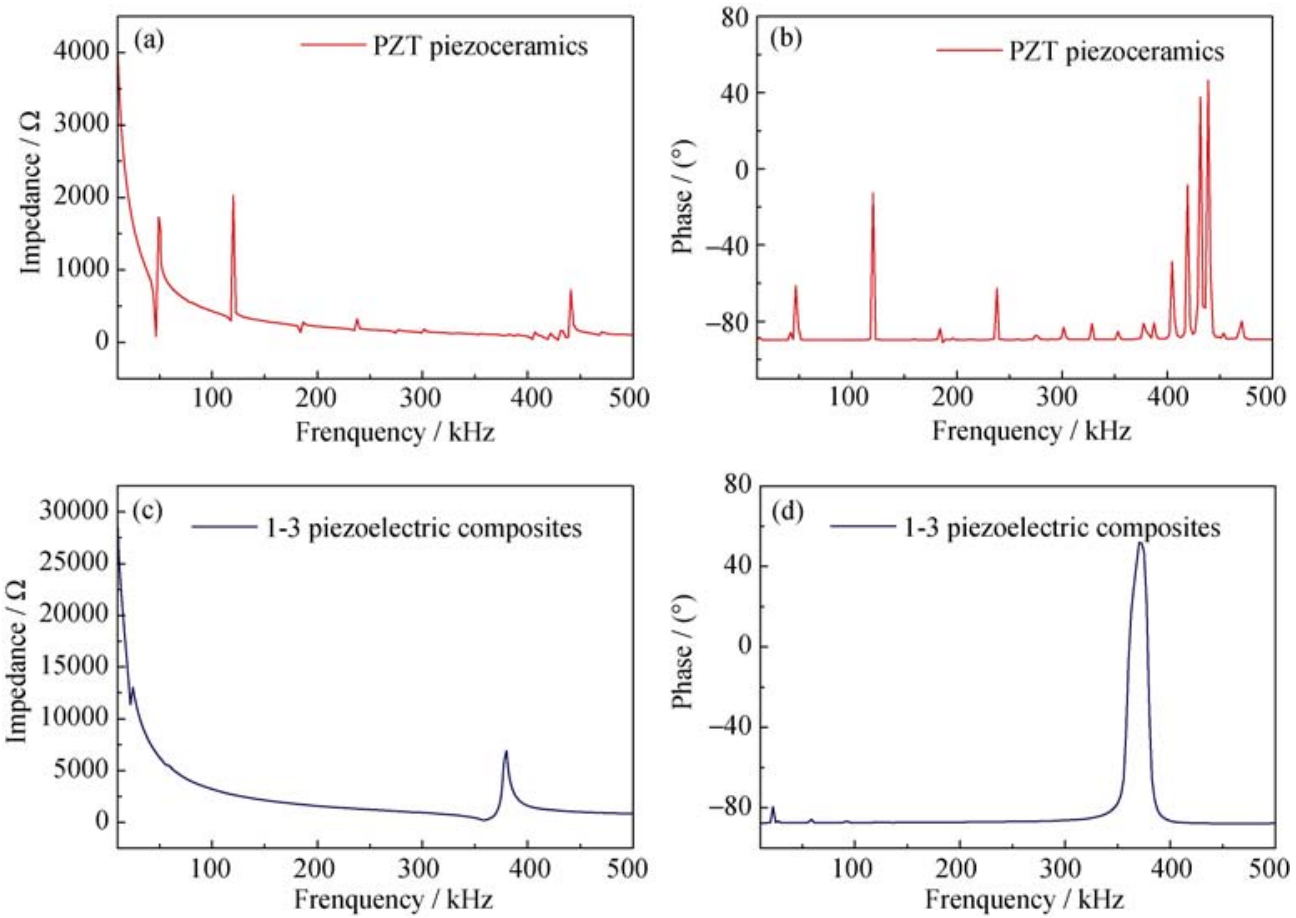

图 5 PZT 压电陶瓷和 1-3 型压电复合材料阻抗谱图

Fig. 5 Frequency-impedance and frequency-phase curves of PZT piezoceramics and 1-3 piezoelectric composites

表 1 材料的机电耦合系数

Table 1 Electromechanical coupling coefficient of material

\begin{tabular}{ccc}
\hline $\begin{array}{c}\text { Material parame- } \\
\text { ters }\end{array}$ & $\begin{array}{c}\text { PZT piezoce- } \\
\text { ramics }\end{array}$ & $\begin{array}{c}\text { 1-3 piezoelectric } \\
\text { composites }\end{array}$ \\
\hline$k_{\mathrm{t}}$ & 0.48 & 0.64 \\
$k_{\mathrm{p}}$ & 0.58 & 0.33 \\
$k_{\mathrm{t}} / k_{\mathrm{p}}$ & 0.83 & 1.94 \\
\hline
\end{tabular}

由于聚焦超声换能器的设计主要是利用厚度振 动模态, 从材料性能分析可知, 采用 1-3 型压电复 合材料, 可以有效提高换能器的电声转换效率。

在此基础上对透镜式聚焦换能器进行了频率特性 测试, 如图 6 所示, 实线是换能器的频率-阻抗特性曲 线, 虚线是换能器的频率-相位特性曲线。对比频率-阻 抗特性曲线可以看到, 1-3 型压电复合材料透镜式聚焦 换能器带宽为 $25 \mathrm{kHz}$, 是 PZT 压电陶瓷透镜式聚焦换 能器的 3.13 倍。另外, PZT 压电陶瓷透镜式聚焦换能器 在测量频率范围 300 600 kHz 内出现较多高幅值的振 动峰, 各种模态耦合现象严重; 而 1-3 型压电复合材料 透镜式聚焦换能器在相同的频率测量范围内呈现单一 的厚度振动模态。由此可见, 1-3 型压电复合材料透镜 式聚焦换能器有效地抑制多模振动耦合现象。

聚焦换能器是利用逆压电效应将电能转化成为 机械能, 声波从压电材料中平行传播出来, 经过粘 结层, 传输至声透镜。声透镜通过会聚作用使波束改 向, 将能量聚集于焦点处 ${ }^{[21]}$ 。研究表明 ${ }^{[22-24]}$, 当 1-3 型压电复合材料中压电陶瓷的体积分数大于 $40 \%$ 时,
其压电常数接近于压电陶瓷的压电常数。因此, 本研 究制作的压电复合材料, 既具有较高的压电常数, 又具有单一的厚度振动模态。

实验采用 OHAUS Adventure ${ }^{\mathrm{TM}}$ Pro 超声功率计 对两种透镜式聚焦换能器进行了声功率测试。图 7(a)、(b)分别为 PZT 压电陶瓷和 1-3 型压电复合材料 透镜式聚焦换能器的输出声功率和电-声功率转换 效率曲线图，从图 7(b)分析可得，基于 PZT 压电陶瓷 的透镜式聚焦换能器电-声转换效率约为 $33.71 \%$, 而 基于 1-3 型压电复合材料的透镜式聚焦换能器电-声 转换效率约为 $63.44 \%$ 。由此可见, 1-3 型压电复合材 料能有效地提高换能器的电声转换效率。

\section{4 结论}

1) 1-3 型压电复合材料可抑制压电陶瓷多种振动模 态耦合现象, 得到较单一的厚度振动模态, 并能有效地 提高换能器的转换效率, 1-3 型压电复合材料透镜式聚 焦换能器是 PZT 压电陶瓷透镜式聚焦换能器的 1.88 倍。

2) 1-3 型压电复合材料透镜式聚焦换能器增加 了换能器的带宽, 达到 PZT 压电陶瓷透镜式聚焦换 能器带宽的 3.13 倍, 更易与水和人体组织相匹配。

在未来高强度聚焦超声(HIFU)换能器应用中, 1-3 型压电复合材料及换能器将成为重要的部分, 它 能改善聚焦超声换能器的宽带及电声转换效率, 提 高高强度聚焦超声治疗设备的可靠性和稳定性。 

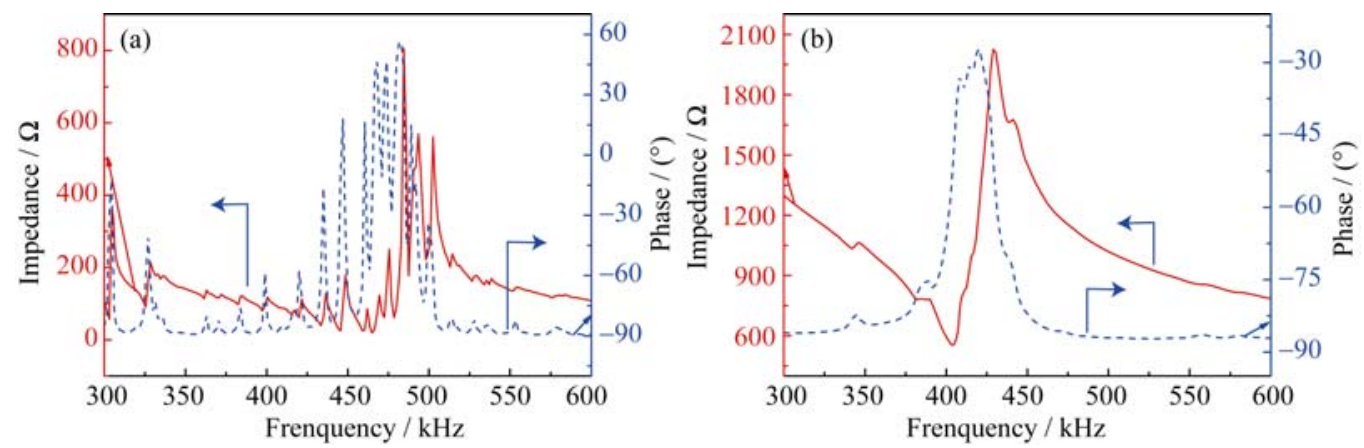

图 6 透镜式聚焦换能器的频率特性曲线图

Fig. 6 Frequency-impedance and frequency-phase curves of lens-focused transducer

(a) PZT piezoceramics; (b) 1-3 piezoelectric composites
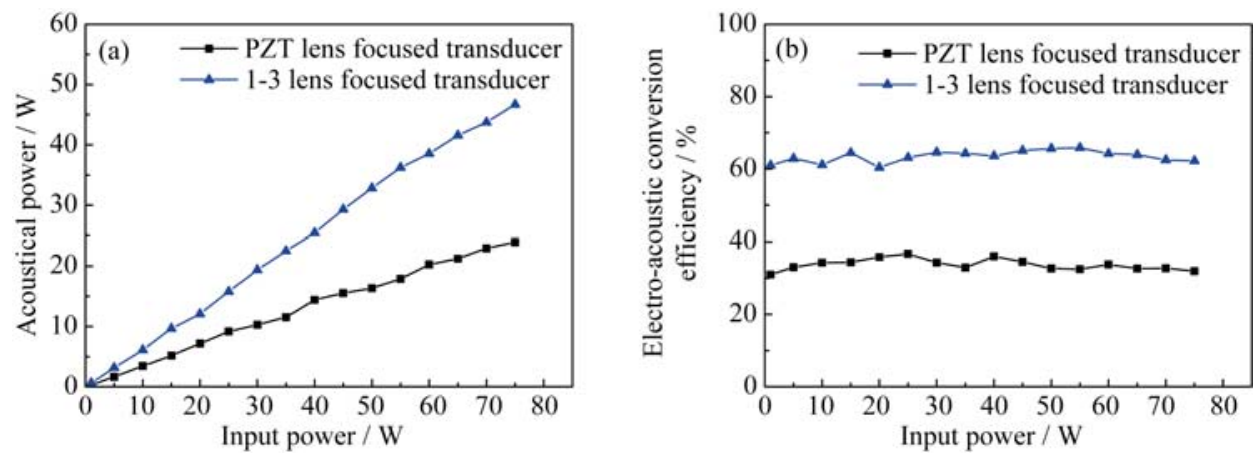

图 7 透镜式聚焦换能器的电声转换曲线

Fig. 7 Electric power-sound power (a) and electro-acoustic conversion efficiency (b) curves of lens-focused transducer

\section{参考文献:}

[1] FENG RUO. Rapid rise of focused ultrasound therapeutic technique and prospect in China. Technical Acoustics, 2011, 30(1): $17-20$.

[2] ZHANG DE-JUN. High intensity focused transducer. Journal of Ultrasonic Diagnosis of China, 2000(1):11-18.

[3] TANG XU-DONG, ZENG DE-PING, YANG ZENG-TAO, et al. Design of the lens-focused transducer based on 1-3 piezoelectric composites. Piezoelectrics \& Acoustiooptics, 2013, 35(4): 546-548.

[4] NEWNHAM R E, SKINNER D P, CROSS L E. Connectivity and piezoelectric-pyroelectric composites. Mat. Res. Bull., 1978, 13(5): 525-536.

[5] WANG SHU-BIN, XU TING-XIAN, HAN JIE-CAI, et al. Preparation and properties of piezoelectric composites of PZT/PVDF. Acta Materiae Compositae Sinica, 2000, 17(4): 1-5.

[6] SOARES CJ, SANTANA F R, PEREIRA J C, et al. Influence of airborne-particle abrasion on mechanical properties and bond strength of carbon/epoxy and glass/bis-GMA fiber-reinforced resin posts. J. Prosthet. Dent., 2008, 99(6): 444-454.

[7] TACK J L, FORD D M. Thermodynamic and mechanical properties of epoxy resin DGEBF crosslinked with DETDA by molecular dynamics. J. Mol. Graph. Model., 2008, 26(8):
$1269-1275$.

[8] ZHANG HUI, ZHANG HUI, TANG LONG-CHENG, et al. The effects of alumina nanofillers on mechanical properties of high-performance epoxy resin. J. Nanosci. Nanotechnol., 2010, 10(11): 7526-7532.

[9] ABRAR A, ZHANG D, SU B, et al. 1-3 connectivity piezoelectric ceramic-polymer composite transducers made with viscous polymer processing for high frequency ultrasound. Ultrasonics, 2004, 42(1-9): 479-484.

[10] HARRIS G R, GAMMELL P M. 1-3 piezoelectric composite transducers for swept-frequency calibration of hydrophones from 100 kHz to 2 MHz. J. Acoust. Soc. Am., 2004, 115(6): 2914-2918.

[11] YANG H C, CANNATA J, WILLIAMS J, et al. Crosstalk reduction for high-frequency linear-array ultrasound transducers using 1-3 piezocomposites with pseudo-random pillars. IEEE Trans. Ultrason Ferroelectr Freq. Control., 2012, 59(10): 2312-2321.

[12] CHEN GIN-SHIN, LIU HSIN-CHIH, LIN YU-LI, et al. Experimental analysis of 1-3 piezocomposites for high-intensity focused ultrasound transducer applications. IEEE Trans. Biomed. Eng., 2013, 60(1): 128-134.

[13] SCHULGASSER K. Relationships between the effective properties of transversely isotropic piezoelectric composite. $J$. 
Mech. Phys. Solids, 1992, 40(2): 473-479.

[14] BENVENISTE Y, DVORAK G J. Uniform fields and universal relations in piezoelectric composites. J. Mech. Phys. Solids, 1992, 40(6): 1295-1312.

[15] DUNN MARTIN L. Micromechanics of coupled electroelastic composites: effevtive thermal expansion and pyroelectric coefficients. J. Appl. Phys, 1993, 73(10): 5131-5140.

[16] JOHN W, SLIWA J R. Method for Making Piezoelectric Composite. US United States Patent. 5239736, 1993.

[17] WANG YUAN-YUAN, LI LI, WANG LI-KUN, et al. Fabrication of 1-3 piezoelectric composite electrode. Functional Materials, 2007, 38(Suppl.): 738-740.

[18] NIEDERHAUSER J J, JAEGER M, FRENZ M. Real-time three-dimensional optoacoustic imaging using an acoustic lens system. Appl. Phys. Lett., 2004, 85(5): 846-848.

[19] ANDREAS HAKANSSON, FRANCISCO CERVERA, JOSE SANCHEZ-DEHESAA. Sound focusing by flat acoustic lenses without negative refraction. Appl. Phys. Lett., 2005, 86(5):
054102-1-3.

[20] GURURAJA $\mathrm{T}$ R, SCHULZE W A, NEWNHAM R E. Piezoelectric composite materials for ultrasonic transducer applications. Part 1: Resonant modes of vibration of PZT rod-polymer composites. IEEE Transactions on Sonic and Ultrasonics, 1985(Suppl.), 32(4): 481-498.

[21] SLIWA JOHN W, GOETZ JOHN P, MA ZHEN-YI, et al. Device for high intensity focused Ultrsound Treatment with Acoustic Lens. U.S. European Patent. 2112906 B1, 2013.

[22] ZHEN ZU-HUA, Li JIN-FENG. Preparation and performance of fine 1-3 type piezoelectric PZT/epoxy resin composite material. Journal of the Chinese Ceramic Society, 2006, 34(3): 381-384.

[23] HUANG SHI-FENG, YE ZHENG-MAO, WANG SHOU-DE, et al. Preparation and properties of 1-3 cement-based piezoelectric composites. Acta Materiae Compositae Sinica, 2007, 24(1): 122-126.

[24] ZHAO SHOU-GEN, CHENG WEI. Research progress of 1-3 type piezoelectric composites. Advances in Mechanics, 2002, 32(1): 57-68. 